\title{
Geometric issues of transforming a topographic surface into a design project.
}

\author{
Dilarom Kuchkarova ${ }^{1 *}$, Dilnoza Achilova ${ }^{2}$ \\ ${ }^{1}$ Tashkent institute of irrigation and agricultural mechanization engineers, Tashkent, Uzbekistan \\ ${ }^{2}$ Belarusian-Uzbek Intersectoral Institute of Applied Technical Qualifications, Tashkent, Uzbekistan
}

\begin{abstract}
Natural relief adjustment for functional purpose - the task of vertical planning - is one of the main complexes of engineering preparation problems of urban areas, industrial sites, reclamation of irrigated land. Numerous methods of designing vertical levelling are aimed at building algorithms that would allow us to obtain an optimal solution in an automated mode. In the set of problems of engineering preparation of urban areas and industrial sites, vertical planning is defined as:

1. a set of engineering and aesthetic measures aimed at adapting the natural relief for the needs of development and subsequent operation, taking into account the functional characteristics of the site

2. a part of engineering preparation, which consists in providing a height arrangement of buildings and structures necessary for the best technological connection between individual objects, as well as a quick collection of atmospheric waters

Reclamation of irrigated lands has specific requirements to design surface maximum preservation of fertile layer, the satisfaction of cultivation technology conditions of different crops and irrigation technique. The main disadvantage of all known methods of designing vertical levelling is a cumbersome solution - a very labor-intensive way of successive approximation to an acceptable solution requires a large expenditure of computer time and subsequent manual revision. The article proposes one of the approaches to the design of levelling using geometric estimates.
\end{abstract}

\section{Introduction}

Modern software packages usually include the following design steps:

1) Determining the design surface elevations that satisfy the specified technical requirements according to the chosen optimality criterion and the specified system of constraints.

2) Selection of excavation contours with the indication of working volumes of cuts and embankments and coordinates of each contour's centers of gravity.

\footnotetext{
* Corresponding author: kuchkarova-dilarom@yandex.ru
} 
3) Selection of the most profitable transportation scheme with the indication of the routes of movement of soil.

4) Calculation of the layout project's overall performance (area of the site, specific volumes of work, areas of work, etc.

5) issuance of design and technical documentation (site plan with an indication of existing, design and working levels, tables of general indicators, tables of the scheme of transportation, etc.)

6) Determination of design elevations of buildings and structures, characteristic points of roads.

7) Graphic representation of the master plan of the object, i.e. layout diagram of buildings, structures and roads with the indication of reference and design elevations for each characteristic point, distances between them, longitudinal slope and its directions.

8) Graphic representation of longitudinal profiles for each road section. [1,3,4,5]

The following parameters are set as input information:

1) Initial elevations, ground and water table category in the nodes of the master plan grid (relief section).

2) Coordinates of planned surface areas on the master plan of the facility.

3) Water flow direction for each planned surface area.

4) Coordinates of buildings, structures, and communications.

5) Limitations on the permitted road slopes.

6) Economic characteristics (cost of soil development, its removal, import). [7,8]

As an economic criterion of optimality is usually considered:

1) The minimum of the total amount of earthworks.

2) Minimum value of cutback, i.e. ensuring maximum proximity of the project to the existing surface.

3) Zero balance of earthworks.

4) Minimum of total costs of land levelling.

5) Minimum cost of construction works $[9,10,19,20]$

The first three goals are the most common in traditional urban planning.

When solving reclamation tasks, the most promising is the criterion of minimum total costs, including the cost of fertility restoration.

Depending on the existing relief complexity and specifics of the problem to be solved, the design surface type may be different.

There are currently software complexes developed in the Republic of Uzbekistan that can satisfy a wide range of designers' requirements - improvement of design quality due to optimization, the possibility to vary design parameters to obtain a more effective technological solution, reduction of design time, etc.

The development of such complexes became possible due to creating the theory and methods of optimal design of vertical planning. [14,15,16,17,18]

Let us note the following moments in the development of the theory and methods of vertical levelling design optimization:

1) During quite a long time, approaches to the analytical solution of vertical planning tasks didn't change essentially - the introduction of modern super and microcomputers into design practice allowed to get a great number of design solutions, to make those or other corrections to the project quickly, to create more comfortable working conditions for designers, but methods of solution of the above tasks remained the same at present as well. 
2) Attempts made by a special selection of target function type and restriction system to solve several optimization problems simultaneously do not lead to the desired result. An overcomplicated task with a large volume of calculations requires the designer mathematical knowledge when necessary to make corrections in the project. In turn, reducing the planning task to subtasks of step-by-step optimization using iterative methods also does not always lead to an optimal solution.

3) The need for "manual" project fine-tuning is due to the lack of an initial data analysis stage, taking into account the terrain's geometric features. Even in cases where software tools allow visualizing the process of creating a layout project, the designer has no reliable means of checking the optimality solution. The vertical layout design strategy is steadily cyclic or branched, while the most effective and cost-effective strategies are linear and with a minimum of cycles.

\section{Methods}

Vertical layout is a multi-model and multi-variant task. Multi-model is due to the differences in the specific conditions and technical specifications in urban planning, industrial and reclamation construction. Multi-variance arises from the availability of multiple design solutions with different costs when the design surface choice, regardless of the type of design, depends on many factors: terms of reference, type of terrain, availability of software and computational tools, etc.

Modern program complexes of planning are oriented at using supercomputers and personal computers of IBM PC type, compatible, depending on the size of the problem to be solved, with AutoCAD system and a wide set of service graphics programs. [2,6]

In the program complexes for vertical planning of industrial areas and sections of urban areas, the method of least squares is used: design marks are determined for characteristic points given within the limits of the master plan so that the sum of the deviations of design marks from the existing ones would be minimal (maximum preservation of relief).

It may be noted that most of the known methods are reduced to the problems of mathematical programming - linear, quadratic, dynamic, etc., most often as follows:

1. Minimize

$$
V=\sum_{j=1}^{n n} \beta_{j \mid h_{j} l}
$$

with conditions $\mathrm{Ah} \geq \mathrm{b}$

where $\mathrm{A}$ is the matrix of coefficients of the system of conditions

$$
\begin{aligned}
\varepsilon_{1} \leq Z_{j}-Z_{j+1} \leq \alpha_{1} \\
\varepsilon_{2} \leq Z_{j}+Z_{j+n} \leq \alpha_{2}
\end{aligned}
$$

$\mathrm{Zj}$ is design elevation, $\mathrm{n}$ is number of marks in a row, $\varepsilon_{1} \alpha-\varepsilon_{1} \alpha-$

$$
\varepsilon_{3} \leq Z_{j+n}-Z_{j+1} \leq \alpha_{3}
$$




$$
\begin{aligned}
& \varepsilon_{4} \leq Z_{j-1}-2 z_{j}+Z_{j+1} \leq \alpha_{4} \\
& \varepsilon_{5} \leq Z_{j-1}-2 z_{j}+Z_{j+n} \leq \alpha_{5}
\end{aligned}
$$

are design parameters.

2. Minimise

$$
Q=h^{T} B h
$$

under the constraints $\mathrm{Ah} \geq \mathrm{b}$, where $\mathrm{A}$ is the matrix of coefficients of the system of conditions of task 1 .

3 Minimise costs

$$
\mathrm{R}=(\mathrm{c}, \mathrm{v})
$$

under the constraints $A h \geq b, B h-D v=O, v \geq 0, C$ is a vector of moving costs per unit of soil along each of the possible routes, $\mathrm{v}$ is a vector of volumes moved along each of possible routes, $\mathrm{B}$ is a diagonal matrix of weights, $\mathrm{D}$ is transport matrix.

4 Minimise the function

$$
V=\sum_{j=1}^{m n} \beta_{j}\left|c-x_{j} i_{x}-y_{j} i_{y}-H_{j}\right|
$$

with restrictions on design slopes

$$
\begin{aligned}
& i_{x}^{\min } \leq i_{x} \leq i_{x}^{\max } \\
& i_{y}^{\min } \leq i_{y} \leq i_{y^{\max }}
\end{aligned}
$$

to the working points

$$
h_{j}^{\min } \leq c-x_{j} i_{x}-y_{j} i_{y}-H_{j}<h_{j}^{\max }
$$

Let us make the following conclusions:

1) It is possible to change the cyclic nature of vertical layout design only by changing the design strategy itself, focusing on analyzing initial data and the existing surface.

2) The search for the design surface should be carried out by setting several optimality criteria, which makes it possible to generate multiple design solutions - this, in turn, is a prerequisite for the functioning of modern design systems.

3) A reliable tool for selecting the optimal solution among the set of obtained solutions is necessary.

4) All variety of variants of design surface search can be reduced to a linear programming problem. [11,12.13] 
The following procedure sequence for vertical planning can be proposed:

1) Analysis of initial data, search and construction of structural lines, regularization of initial data network.

2) Determination of interpolant type, construction of interpolating (approximating) surface.

3) Partitioning the regions into sections Gi with equal curvature (into sections of equal complexity). One of the possible partitioning options is shown in Fig. 1

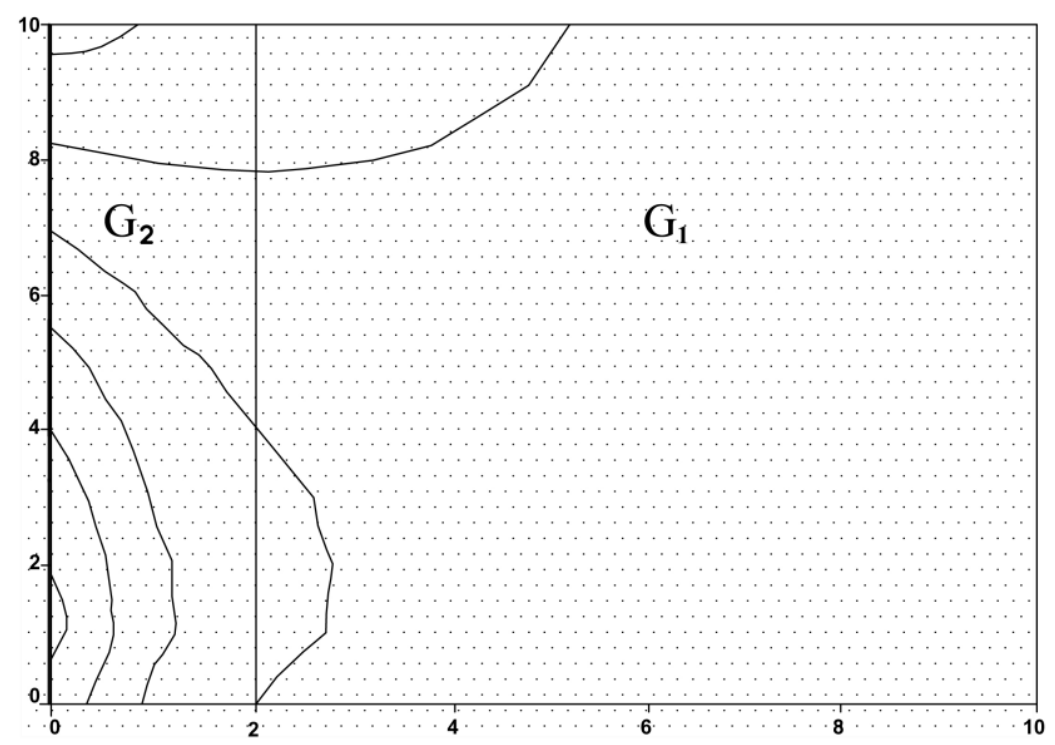

Fig. 1

1) Determination of preliminary geometric estimates. The known vertical levelling design methods do not include this step, but geometric estimates more fully characterize both the existing and the projected surface.

2) It is also possible to estimate $G$ of positive, negative, and mixed curvature with known Ri (Fig. 1)

3) Selection of the design surface, depending on the design situation (under a plane, under a plane system, under a two-slope plane, under a topographic surface, etc.)

4) Selection of the target function, followed by the solution of the linear programming problem.

5) Solution of the transport problem between the planned sites $\mathrm{Gi}$

6) Selection of the design solution among the set of generated ones. 


\section{Results}

Consider the following example. Let the relief section be given, represented by the DEM

$\begin{array}{cccccccccc}6.667 & 6.417 & 6.5 & 6.717 & 7 & 7.321 & 7.667 & 8.028 & 8.4 & 8.78 \\ 7.583 & 7.389 & 7.417 & 7.533 & 7.694 & 7.881 & 8.083 & 8.296 & 8.517 & 8.742 \\ 8 & 7.833 & 7.833 & 7.9 & 8 & 8.119 & 8.25 & 8.389 & 8.533 & 8.682 \\ 8.217 & 8.067 & 8.05 & 8.087 & 8.15 & 8.229 & 8.317 & 8.411 & 8.51 & 8.612 \\ 8.333 & 8.194 & 8.167 & 8.183 & 8.222 & 8.274 & 8.333 & 8.398 & 8.467 & 8.538 \\ {[A]=8.393} & 8.262 & 8.226 & 8.229 & 8.25 & 8.282 & 8.321 & 8.365 & 8.412 & 8.461 \\ 8.417 & 8.292 & 8.25 & 8.242 & 8.25 & 8.268 & 8.292 & 8.319 & 8.35 & 8.383 \\ 8.417 & 8.296 & 8.25 & 8.233 & 8.231 & 8.238 & 8.25 & 8.265 & 8.283 & 8.303 \\ 8.4 & 8.283 & 8.233 & 8.21 & 8.2 & 8.198 & 8.2 & 8.206 & 8.213 & 8.223 \\ 8.371 & 8.258 & 8.205 & 8.176 & 8.159 & 8.149 & 8.144 & 8.141 & 8.141 & 8.142 \\ 8.333 & 8.222 & 8.167 & 8.133 & 8.111 & 8.095 & 8.083 & 8.074 & 8.067 & 8.061\end{array}$

structural lines and structural model of the relief area are determined. The figure shows the level lines of the approximation surface, the areas with uniform curvature are marked, the radii of the maximum neighborhoods are given.

Preliminary geometrical estimates for plots $\mathrm{G}_{1}$ and $\mathrm{G}_{2}$ are determined

The radii of the maximum neighborhoods of the sections $G_{1}$ and $G_{2}$ are given 6 c.u. (conventional unit) and 20 c.u. (conventional unit) respectively depending on the scale. the length of the boundary of the region $G_{1}$

$$
\begin{aligned}
P_{n} \approx 20[3 \pi+ & \left.\frac{(36-14-3) \pi}{3}-(36-20) W_{(4)}^{(+)+}\right]=20 \cdot\left[3 \cdot 3,14+\frac{19 \cdot 3,14}{3}-16 \cdot 1\right] \\
& =266,3 \text { c.u. }
\end{aligned}
$$

the length of the boundary of the region $G_{2}$

$$
P_{n} \approx 6\left[3 \pi+\frac{6 \cdot \pi}{3}+9 \cdot W_{(4)}^{(1)^{*}}\right] \quad P_{n} \approx 6\left[3 \pi+\frac{6 \cdot \pi}{3}+9 \cdot 1\right]=148,2 \text { c.u. }
$$

preliminary estimates of areas $G_{1}$ and $G_{2}$, respectively

$$
\begin{gathered}
S_{1} \leq \frac{C R^{2}\left(2 \pi-W_{(4) i}^{(1) *}\right)^{2}}{4 \pi}=\frac{2 \cdot 400(2 \cdot 3,14-1)^{2}}{4 \cdot 3,14}=1775,6(\text { squares c.u. }) \\
S_{2} \leq \frac{4 \cdot 36(2 \cdot 3,14+1)^{2}}{4 \cdot 3,14}=607,6(\text { squares c.u. })
\end{gathered}
$$

Consider several options for constructing a design surface represented by a system of planes. In the case when the goal function is the minimum of the total volume of earthworks, the discrete analogue of the desired model of the design surface will be determined as a result of solving the linear programming problem: (for section $G_{1}$ ) minimize the objective function 


$$
V=\sum_{\substack{i=1 \\ j=1 \\ j=1}}^{\substack{i=4 \\ i j}} z_{i j} \rightarrow \min \quad z_{i j}=\frac{z_{1 j}+z_{2 j}+z_{1 j+1}+z_{2 j+1}}{4}
$$

$S_{i j}$ is area of the $\mathrm{j}$-th network element

$$
\begin{aligned}
& V_{1}=S_{1} z_{1}^{(c)}+S_{2} z_{2}^{(c)}+\cdots+S_{38} z_{38}^{(c)} \rightarrow \min \\
& S_{1}=S_{37}=8,02 \quad S_{2}=S_{38}=7,94 \quad S_{3}=S_{36}=7,96 \quad S_{4}=S_{35}=8,03 \\
& S_{5}=S_{34}=8,24 \quad S_{6}=S_{33}=8,12 \quad S_{7}=S_{32}=8,10 \quad S_{8}=S_{31}=8,14 \\
& S_{9}=S_{30}=8,34 \quad S_{10}=S_{29}=8,22 \quad S_{11}=S_{28}=8,27 \quad S_{12}=S_{27}=8,24 \\
& S_{13}=S_{26}=8,33 \quad S_{14}=S_{25}=8,21 \quad S_{15}=S_{24}=8,01 \quad S_{16}=S_{23}=7,96 \\
& S_{17}=S_{22}=8,03 \quad S_{18}=S_{21}=7,93 \quad S_{19}=S_{20}=7,98
\end{aligned}
$$

under the following restrictions

$$
\left\{\begin{array}{c}
2 Z_{1}^{(C)}+2 Z_{2}^{(C)}+Z_{3}^{(C)}+Z_{4}^{(C)}+Z_{5}^{(C)}+2 Z_{6}^{(C)}+Z_{7}^{(C)}+Z_{8}^{(C)}+Z_{9}^{(C)}+Z_{10}^{(C)}+Z_{11}^{(C)}+Z_{12}^{(C)}+ \\
+Z_{13}^{(c)}+Z_{14}^{(c)} \geq 120,7 \\
Z_{15}+Z_{16}+2 Z_{17}+2 Z_{18}+Z_{19}+Z_{20}+Z_{21}+Z_{22}+Z_{23}+Z_{24}+Z_{25}+Z_{26}+Z_{27} \geq 106,5 \\
2 Z_{1}+Z_{2}+Z_{3}+2 Z_{28}+Z_{29}+Z_{30}+Z_{31}+Z_{32}+Z_{33}+Z_{34}+Z_{35}+Z_{36}+Z_{37}+Z_{38} \geq 113,6 \\
2 Z_{1}+Z_{2}+Z_{3}+Z_{4}+Z_{5}+2 Z_{6}+Z_{7}+Z_{8}+Z_{9}+Z_{10}+Z_{11}+Z_{12}+Z_{13}+Z_{14} \leq 128,4 \\
2 Z_{15}+2 Z_{16}+Z_{17}+Z_{18}+Z_{19}+Z_{20}+Z_{21}+Z_{22}+Z_{23}+Z_{24}+Z_{25}+Z_{26}+3 Z_{27} \leq 138,6 \\
2 Z_{28}+Z_{29}+Z_{30}+Z_{31}+Z_{32}+Z_{33}+Z_{34}+Z_{35}+Z_{36}+Z_{37}+2 Z_{38} \leq 107,8 \\
Z_{1}+3 Z_{2}+2 Z_{3}+Z_{4}+Z_{5}+Z_{6}+Z_{7}+Z_{8}+Z_{9}+Z_{10}+Z_{11}+Z_{12}+Z_{13}+Z_{14}=124,78 \\
3 Z_{15}+Z_{16}+Z_{17}+Z_{18}+Z_{19}+Z_{20}+Z_{21}+Z_{22}+Z_{23}+Z_{24}+Z_{25}+Z_{26}+Z_{27}+Z_{28}=127,54 \\
Z_{1}>0 \quad Z_{2}>0 \quad \ldots \quad Z_{38}>0
\end{array}\right.
$$

for the $G_{2}$ section, a similar problem of minimizing

$$
\begin{aligned}
& V_{2}=S_{1}^{\prime} Z_{1}^{\prime(c)}+S_{2}^{\prime} Z_{2}^{\prime(c)}+\cdots+S_{9}^{\prime} Z_{9}^{\prime} \rightarrow \min \\
& \begin{array}{ccccc}
S_{1}^{\prime}=7,00 & S_{2}^{\prime}=6,92 & S_{3}^{\prime}=7,03 & S_{4}^{\prime}=7,24 & S_{5}^{\prime}=7,47 \\
& S_{6}^{\prime}=7,73 & S_{7}^{\prime}=8,01 & S_{8}^{\prime}=8,31 & S_{9}^{\prime}=8,60
\end{array}
\end{aligned}
$$

under the following restrictions

$$
\left\{\begin{array}{c}
2 Z_{1}^{\prime}+Z_{2}^{\prime}+Z_{3}^{\prime}+Z_{4}^{\prime}+Z_{5}^{\prime}+Z_{6}^{\prime}+Z_{7}^{\prime}+Z_{8}^{\prime}+2 Z_{9}^{\prime} \geq 83,24 \\
Z_{1}^{\prime}+2 Z_{2}^{\prime}+Z_{3}^{\prime}+2 Z_{4}^{\prime}+Z_{5}^{\prime}+Z_{6}^{\prime}+Z_{7}^{\prime}+Z_{8}^{\prime}+Z_{9}^{\prime} \leq 86,16 \\
Z_{1}^{\prime}+2 Z_{2}^{\prime}+4 Z_{3}^{\prime}+Z_{4}^{\prime}+Z_{5}^{\prime}+Z_{6}^{\prime}+Z_{7}^{\prime}+Z_{8}^{\prime}+2 Z_{9}^{\prime}=106,41 \\
Z_{1}^{\prime}>0 \quad Z_{2}^{\prime}>0 \ldots Z_{9}^{\prime}>0
\end{array}\right.
$$

As a result of the implementation of the simplex-algorithm, we obtain the following values of heights for sections $G_{1}$ and $G_{2}$

$$
\begin{array}{ccccc}
Z_{1}^{(c)}=7,1 & Z_{2}^{(c)}=7,3 & Z_{3}^{(c)}=7,0 & Z_{4}^{(c)}=7,1 & Z_{5}^{(c)}=7,0 \\
& & Z_{6}^{(c)}=7,1 & Z_{7}^{(c)}=7,0 & \\
Z_{8}^{(c)}=7,8 & Z_{9}^{(c)}=7,2 & Z_{10}^{(c)}=7,3 & Z_{11}^{(c)}=7,2 & Z_{12}^{(c)}=7,1 \\
& & Z_{13}^{(c)}=7,2 & Z_{14}^{(c)}=7,1 &
\end{array}
$$




$$
\begin{array}{ccccc}
Z_{15}^{(c)}=7,2 & Z_{16}^{(c)}=7,2 & Z_{17}^{(c)}=7,3 & Z_{18}^{(c)}=7,5 & Z_{19}^{(c)}=7,4 \\
Z_{22}^{(c)}=7,2 & Z_{23}^{(c)}=7,3 & Z_{24}^{(c)}=7,1 & Z_{21}^{(c)}=7,2 & \\
& Z_{27}^{(c)}=7,2 & Z_{28}^{(c)}=7,1 & Z_{25}^{(c)}=7,3 \\
Z_{29}^{(c)}=7,1 & Z_{30}^{(c)}=7,1 & Z_{31}^{(c)}=7,4 & Z_{32}^{(c)}=7,04 \\
& Z_{34}^{(c)}=7,2 & Z_{33}^{(c)}=7,1 \\
Z_{1}^{\prime}=6,13 & Z_{35}^{(c)}=Z_{36}^{(c)}=Z_{37}^{(c)}=Z_{38}^{(c)}=7,2 & \\
& Z_{2}^{\prime}=6,24 & Z_{3}^{\prime}=6,31 & Z_{4}^{\prime}=6,34 & Z_{5}^{\prime}=6,13 \\
& Z_{6}^{\prime}=6,423 & Z_{7}^{\prime}=6,43 & Z_{8}^{\prime}=Z_{9}^{\prime}=6,37 &
\end{array}
$$

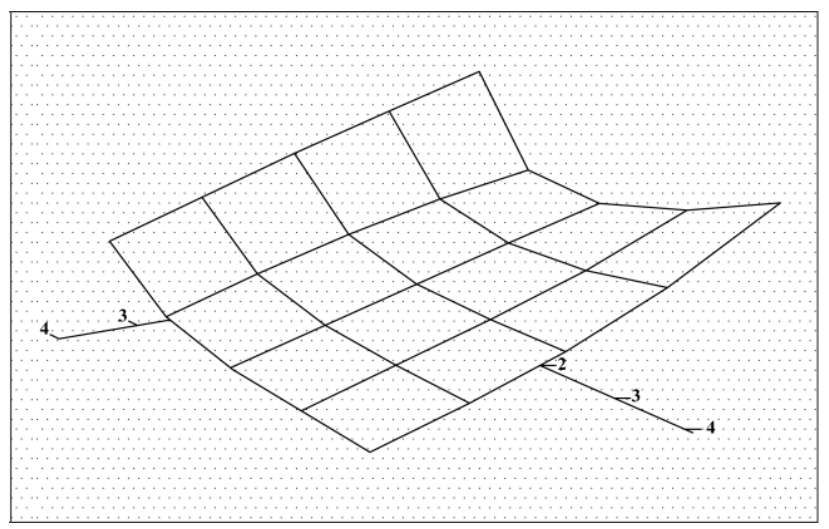

Fig. 2

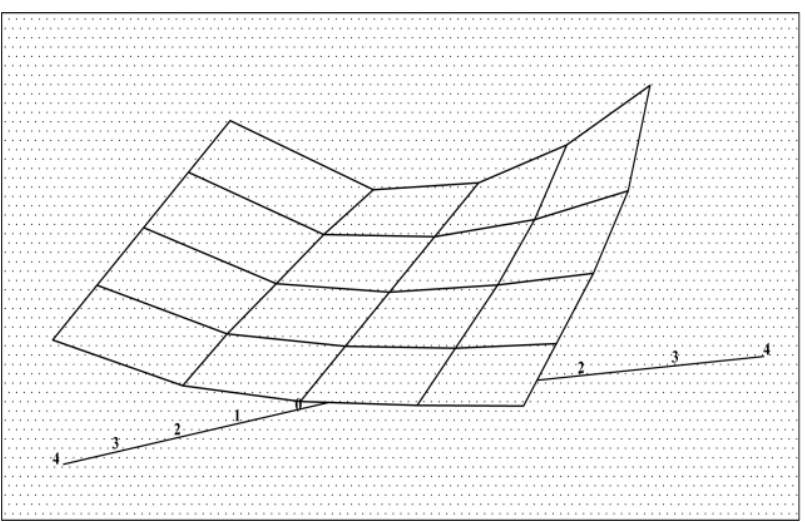

Fig. 3

At this stage, the required surface is represented by figures 1 and 2 . If the systems of restrictions and additional conditions are set - the maximum preservation of the existing relief, that is, to limit the difference $\Delta Z_{i}$ between the heights of the design and existing surface to $\Delta Z_{i}=0,2$, then the sought surface will be represented by the fig. 4 


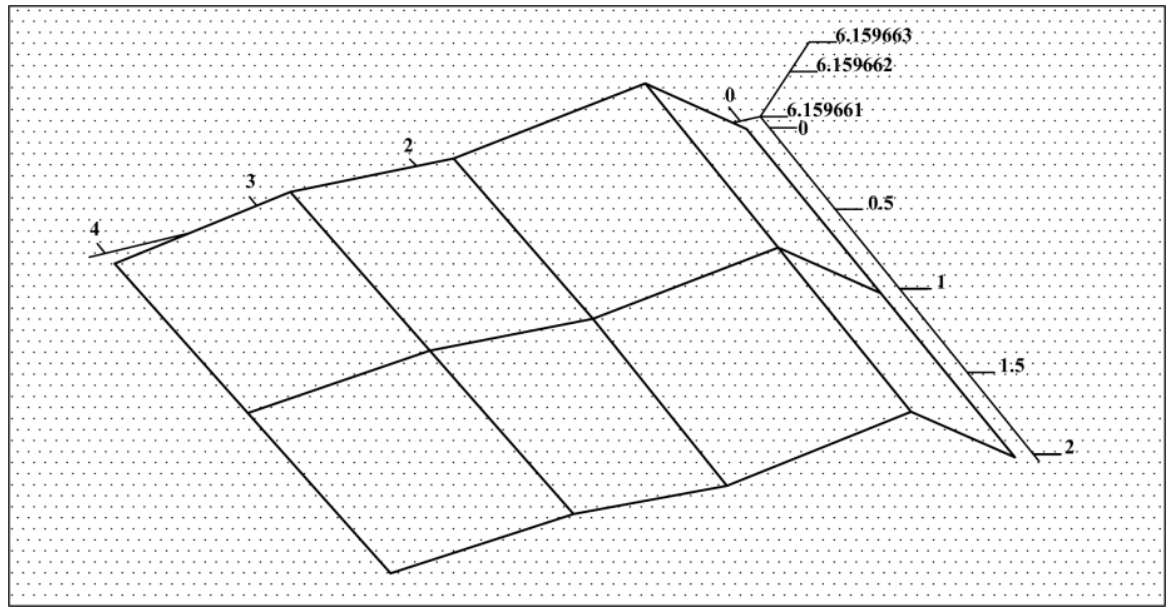

Fig. 4

at the next stage, between the planned sections $G_{1}$ and $G_{2}$, the transport problem is solved:

$$
\sum_{i=1}^{5} \sum_{j=1}^{9} C_{i j} X_{i j} \rightarrow \min
$$

Where $X_{i j}$ is the amount of soil transported from the $\mathrm{i}$ section to the $\mathrm{j}$ $C_{i j}$-the cost of transporting soil from the $\mathrm{i}$ section to the $\mathrm{j}$

The resulting surface is shown in Fig. 5

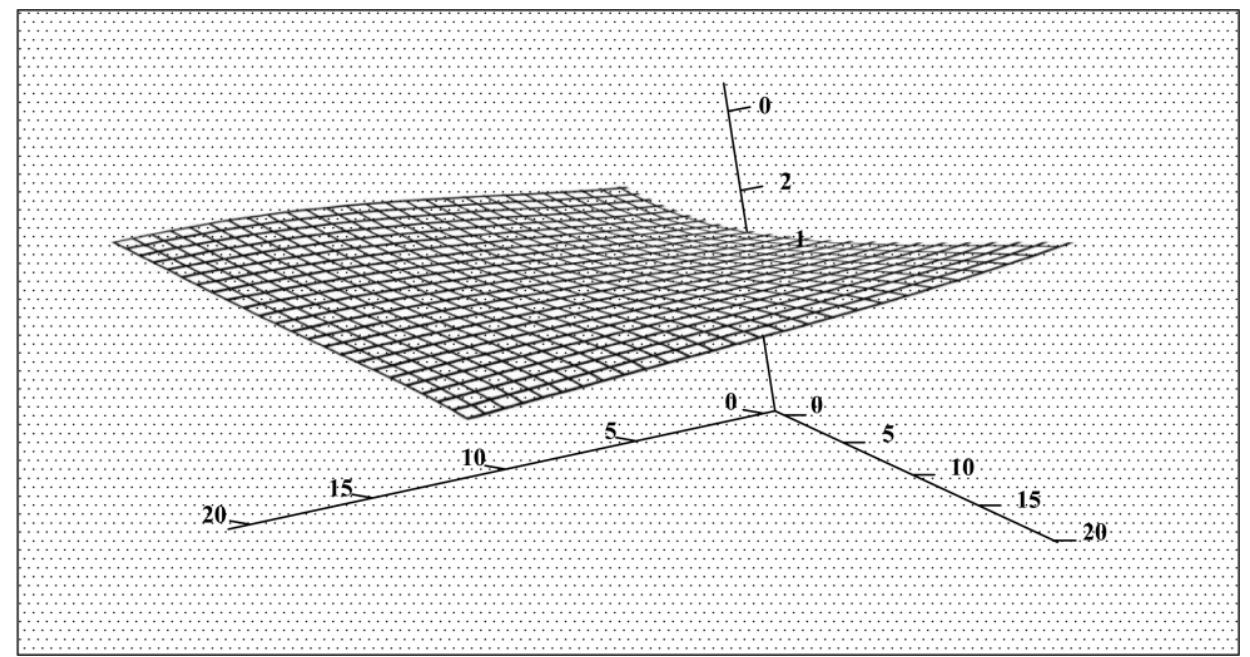

Fig. 5

In specifying a system of constraints, the goal function takes the value $f_{\min }=2292,36$ (cube c.u.) When the system of restrictions changes, the target function also changes $f_{\min }=1892,36$ (cube c.u.)

\section{Conclusion}


The vertical levelling task, being geometric in essence, requires geometric methods for its solution, not only mathematical ones.

Preliminary analysis of the existing surface, areas of varying curvature allows you to give an advance estimate of the upcoming layout.

This, in turn, reduces the number of cycles in the design process.

Geometric estimates of areas with equal curvature also reduce the time of solving the transport issue.

\section{References}

1. Akhremenko S. A. Features of urban planning. Tutorial M.: Association of Construction Universities, p.521, (2014)

2. All higher mathematics. Vol 6. Calculus of variations, linear programming, computational mathematics, spline theory. - M.: Librocom, - p.256, (2013)

3. Bakutis V.E. Engineering preparation of urban areas M.: High school, p.138, (2016)

4. Beyerbakh B.A. Engineering networks, preparation of territories and buildings M.: Phenix, p.576, (2009)

5. Chernikh A.G. International design normative base (Eurocodes). Short course of lectures. Tutorial M.: Publishing House of the Association of Building Universities, p.306, (2015)

6. Demidova L.A. Algorithms and systems of fuzzy inference when solving problems of diagnostics of Urban engineering communications in Matlab: monograph. M.:Radio and communication, p.368, (2005)

7. Engineering protection of territories, buildings and structures from hazardous geological processes. Basics of the position. SNiP 22-02-2003. - M.: ДЕAH, p.284, (2005)

8. Gayevoy A.F. Course and diploma design. Industrial and civil buildings. Tutorial M: Alyans, p.264, (2014)

9. Gavrilov D. A. Design and estimate business M.:Alpha-M, p.352, (2014)

10. Komkov B.A. Technical maintenance of buildings and structures M.: ИNFRA-M379, (2017)

11. Kuchkarova, D.F., Achilova, D.A. Identification method of topographic surfaces models, Advances in Intelligent Systems and Computing, ICGG-2018, 809, pp.842-848, (2019)

12. Kuchkarova, D.F., Achilova, D.A. Morphisms general properties of geometrical models, Journal of Physics: Conference Series, Omsk- 2019, 1260(7), 072011.

13. Kuchkarova, D., Achilova, D. Algorithms for choosing a design solution for transforming a relief IOP Conference Series: Materials Science and Engineering, 2020, 883(1), 012022.

14. Kliorina G.I. Engineering preparation of urban areas 2nd ed., Rev. and add. Academic Baccalaureate Textbook M.: Youright, P.559, (2017)

15. Nikolayevskaya I.A. Engineering networks and equipment of territories, buildings and construction sites M.: Academia, p.224, (2004)

16. On the Reclamation and Protection of Agricultural Land p.94, (2012), ISBN: 1290823073.

17. Rijikov I.B. Fundamentals of engineering surveys in construction. Tutorial M.: Lan, - p.136, (2016)

18. Shukhurov I. S. Urban planning, planning of rural settlements. Tutorial. M: Science - p.917, (2016)

19. Shirshikov B.F. Development of construction plans. Design Tutorial M.: ACB, - p.688, (2012)

20. Vorobyov V. S. Construction Infrastructure Management Industrial and civil construction №11 p.49, (2009) 